\title{
Effect of heat treatment on fatigue crack initiation of laser powder bed fusion stainless steel 316 L
}

\author{
Meng Zhang ${ }^{1}$, Chen-Nan Sun ${ }^{2}$, Xiang Zhang ${ }^{3}$, Phoi Chin $\mathrm{Goh}^{4}$, Jun Wei ${ }^{2}$, David Hardacre ${ }^{4}$, and Hua $\mathrm{Li}^{1, *}$ \\ ${ }^{1}$ Singapore Centre for 3D Printing, School of Mechanical and Aerospace Engineering, Nanyang Technological University, 50 \\ Nanyang Avenue, Singapore 639798 \\ ${ }^{2}$ Singapore Institute of Manufacturing Technology, ASTAR, 73 Nanyang Drive, Singapore 637662 \\ ${ }^{3}$ Faculty of Engineering, Environment \& Computing, Coventry University, Coventry CV1 5FB, UK \\ ${ }^{4}$ Lloyd's Register Global Technology Centre, 1 Fusionopolis Place, \#09-11 Galaxis, Singapore 13852
}

\begin{abstract}
Laser powder bed fusion (L-PBF) is an emerging additive manufacturing technique for building structural components. L-PBF processing defects, such as lack of fusion pores, promote fatigue crack initiation and shorten the fatigue life. With well-controlled processing, critical pores can be avoided such that the microstructure-driven intergranular crack initiation mode becomes operative. In this work, the fatigue crack initiation behaviours of as-built and solution annealed L-PBF stainless steel 316L were studied. Crack initiation of the as-built samples is driven by de-bonding of the dendritic grain boundaries. High temperature annealing results in the formation of thermally-induced defects, possibly via the reheat cracking mechanism and the nucleation of pre-existing gas pores. As heat treating could have led to recrystallization and annihilation of the original grain boundary defects, the thermally-induced defects became the new sites for crack initiation. In addition, heat treatment incurred significant reduction in yield strength, such that the interaction of fatigue and ratcheting strain accumulation dominated the deformation behaviour of the material. The resulting fatigue strength in the finite life regime was reduced by about $13 \%$ but the fatigue endurance limit was not affected.
\end{abstract}

\section{Introduction}

In laser powder bed fusion (L-PBF) processing, parts are built layer-by-layer via the fusion of metal powder by high power laser. Laser movements are controlled based on input from computer-aided design models such that near-net-shape parts could be produced. Such design and manufacturing flexibility makes L-PBF the ideal platform for various creative applications, including high performance lattice structures [1], sophisticated components such as injection moulding tools [2] and novel heat transfer devices [3], as well as customised medical products $[4,5]$.

The implementation of this process for actual part production is met with difficulties rooted in the complex processing-structure-property relations. The L-PBF technique is affected by a large number of variabilities, including laser settings, power characteristics, build orientations etc., all of which could affect the evolution of microstructure, trigger the formation of processingdefects and impact the mechanical performance [6-8]. Considering the current lack of field experience with additive manufactured parts and appropriate nondestructive inspection method for detecting micron-size defects [9], questions remain in the integrity and reliability of L-PBF components, especially if they were to be used for safety-critical applications.

For conventionally-manufactured parts, such as welded [10] and cast [11] structures, heat treatment is often employed as the remediation measure for promoting uniformity of composition and microstructure, and to prevent unexpected part failure. The applicability of this approach to L-PBF needs to be examined in relation to the nature of the process-induced microstructure and defects.

For instance, as a result of rapid cooling, high residual stress, exceeding the material's yield strength, had been observed in as-built L-PBF materials [12]. Local plastic deformation could lead to geometrical distortion and cracking [13]. Besides preheating the build platform for reducing the thermal gradient during processing [14], post-processing stress-relieving heat treatment [15] could also be performed to ameliorate the residual stress. Such procedures had been shown to be effective in improving the fracture toughness of Ti6Al4V [16] and the tensile properties of a nickel-base superalloy [17].

Another key processing issue is porosity. Deviations from the optimum build settings and poor process maintenance, e.g. laser miscalibration or the use of damaged recoater blade, could affect the energy available for consolidating the metal powder and promote the formation of lack of fusion defects. As these defects are formed unintentionally and thereby difficult to be controlled, they remain in the as-built parts and increase the risk of component failure. Results from experimental fatigue test had shown that un-melted regions are the primary cause for fatigue crack initiation

Corresponding author: lihua@ntu.edu.sg 
and degraded fatigue strength [18-20]. In response, hot isostatic pressing (HIP), which involves the simultaneous application of heat and pressure, could be employed for removing residual porosity. The fatigue resistance of materials such as TiAl6V4 [21, 22] was found to improve after HIP because of the elimination of failure-critical lack of fusion defects. However, materials with complex microstructures, e.g. Inconel 718 [23] and maraging steel [24], could be prone to failure from second phase particles, and thereby require tailored postprocessing heat treatments.

Stainless steel 316L is one of the highly investigated alloys that showed good manufacturability via the LPBF process. Its fatigue properties are of general interest because of the material's suitability for various fatiguerelated applications, e.g. biomedical devices [25]. Prior works on this material showed that its fatigue resistance is not significantly altered by low temperature stressrelieving heat treatment [26, 27]. To further understand the influence and applicability of heat treatment, higher temperature annealing was performed in this work for examining the effect of more pronounced changes in microstructure on the fatigue failure behaviour of this alloy.

\section{Experimental setup}

Samples were built on a L-PBF machine (EOS M290) equipped with a $400 \mathrm{~W}$ Yb-fiber laser. Commercial stainless steel $316 \mathrm{~L}$ powder with a predominantly spherical shape was used as the raw material for processing. L-PBF build parameters were optimised for a layer thickness of $20 \mu \mathrm{m}$ and built on a preheated platform.

As shown in Figure 1a, samples were fabricated as rectangular blocks, with the long edge being oriented at a small angle from the recoating direction to prevent uneven powder deposition due to interaction between the build part and the recoater blade [28]. After being removed from the baseplate, the blocks were machined

(a)

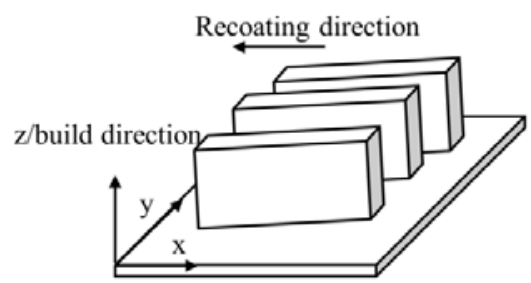

(b)

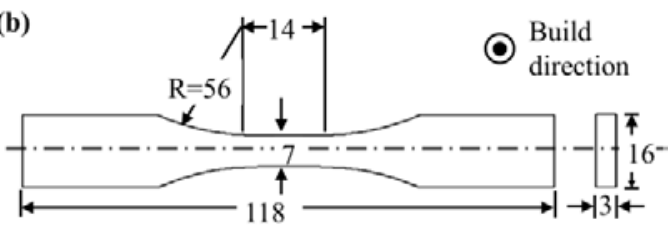

Fig. 1. (a) Orientation of rectangular blocks on the L-PBF build platform. (b) Geometry of the fatigue test specimen. Eight test specimens were machined from each rectangular block by wire cutting. into test specimen geometry by electrical discharge machining (EDM) wire cutting. Eight specimens were sliced from each block along the height such that the loading directions of the specimens are perpendicular to the build direction. Flat fatigue test specimens were designed according to requirements of the ASTM E466 and the dimensions are as shown in Figure 1b.

Some as-built specimens were subjected to annealing heat treatment in accordance with AMS2750 at a holding temperature of $1093{ }^{\circ} \mathrm{C}$ for 25 mins in a vacuum furnace and subsequently gas quenched. Final grinding step was performed prior to mechanical testing to remove any surface contaminants that could have been generated from the heat treatment.

Load-controlled fatigue tests were performed by applying sinusoidal loading at frequency of $5 \mathrm{~Hz}$ and load ratio of 0.1 on an MTS 810 hydraulic testing machine under ambient condition. Specimens that did not fail after $10^{6}$ cycles were considered as runouts. For microstructural analysis, specimen cross sections parallel to the build direction were polished and observed using optical microscope. Scanning electron microscope (SEM) was employed for examination of the fracture surfaces.

\section{Results and discussion}

\subsection{Crack-initiating defects}

SEM fracture images of the as-built and heat-treated samples are displayed in Figure 2, where the crack initiation zones are marked by dotted lines. The as-built sample is characterised by intergranular crack initiation, where fine solidification dendrites, or sub-grains, are clearly shown in Figure 2b. Cracking of this nature is indicative of intergranular brittleness which, for L-PBF stainless steel $316 \mathrm{~L}$, could be attributed to the presence of high dislocation density and elemental segregation at grain boundaries. Specifically, studies done by Zhong et al. [29] had shown that the cellular boundaries are enriched with molybdenum atoms and nano-sized inclusions, which could create local stress concentration and promote cracking. Details of the fatigue fracture mechanisms for as-built L-PBF stainless steel 316L could be found in the authors' prior work [30].

A different type of defect was observed at the crack origin of the heat-treated sample (Figure 2c). The defect adopts a flat and 'compressed' appearance; the distinct line marking the boundary between the defect and the crack propagation region indicates that the defect could be pre-existing in the material after heat treatment.

To further analyse the origins of the defects, optical micrographs of the samples were taken, as shown in Figure 3. For the as-built condition (Figure 3a), small isolated pores with equivalent circular diameters on the order of $5 \mu \mathrm{m}$ are present. Pores of this nature are unavoidable for the L-PBF process due to the entrapment of gas bubbles, which could have originated from the evaporation of low melting point elements or oxygen gas pre-existing in the powder feedstock [31]. Relative to the grain boundary defects in Figure $2 b$, such process- 

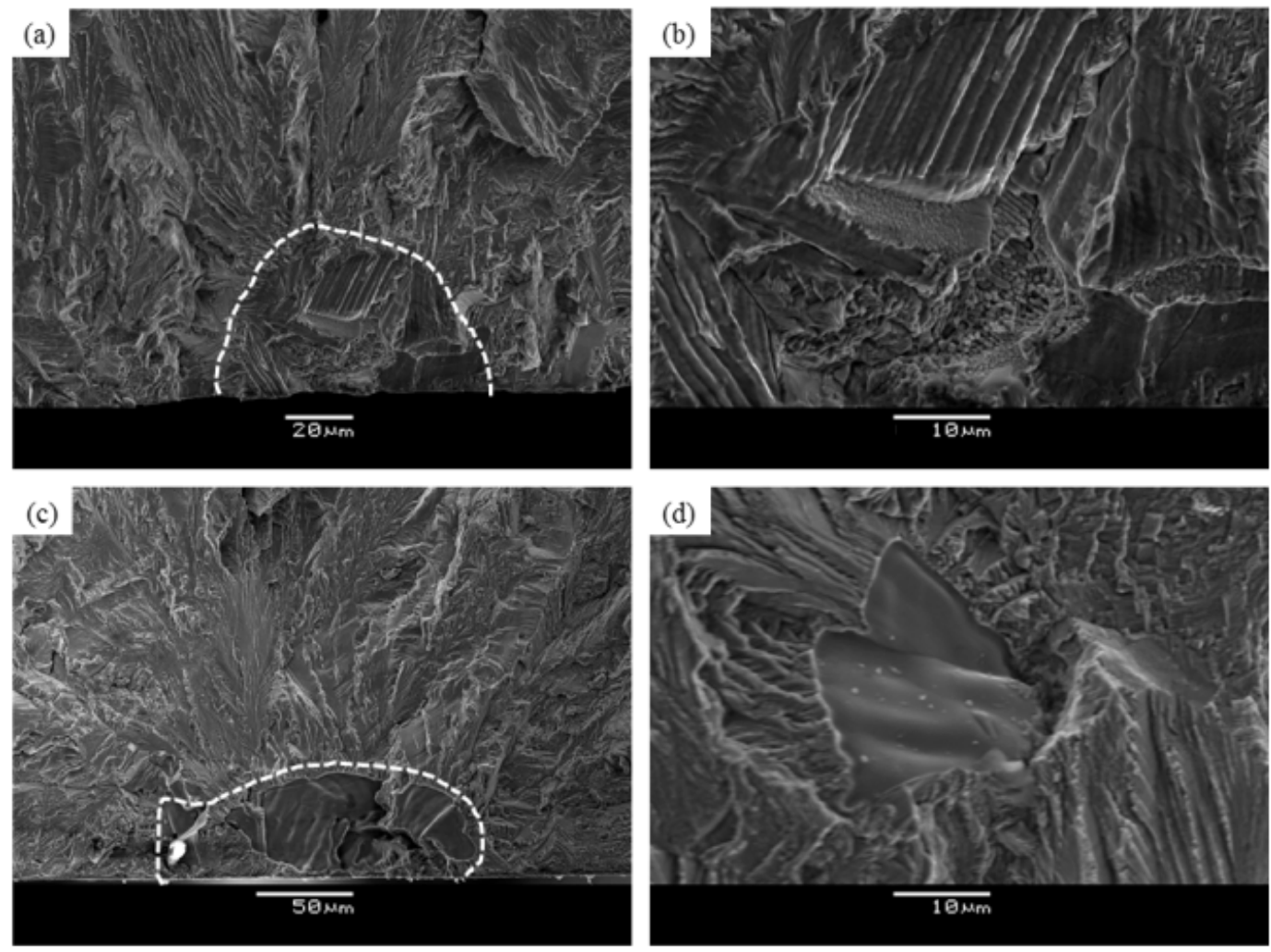

Fig. 2. SEM fracture images of samples subjected to cyclic loads. (a) Crack initiation region (dotted line) of as-built sample; (b) enlarged view of (a) showing solidification dendrites revealed by intergranular cracking; (c) crack initiation region of a heat-treated sample; (d) similar but smaller defect in the interior of a heat-treated sample.
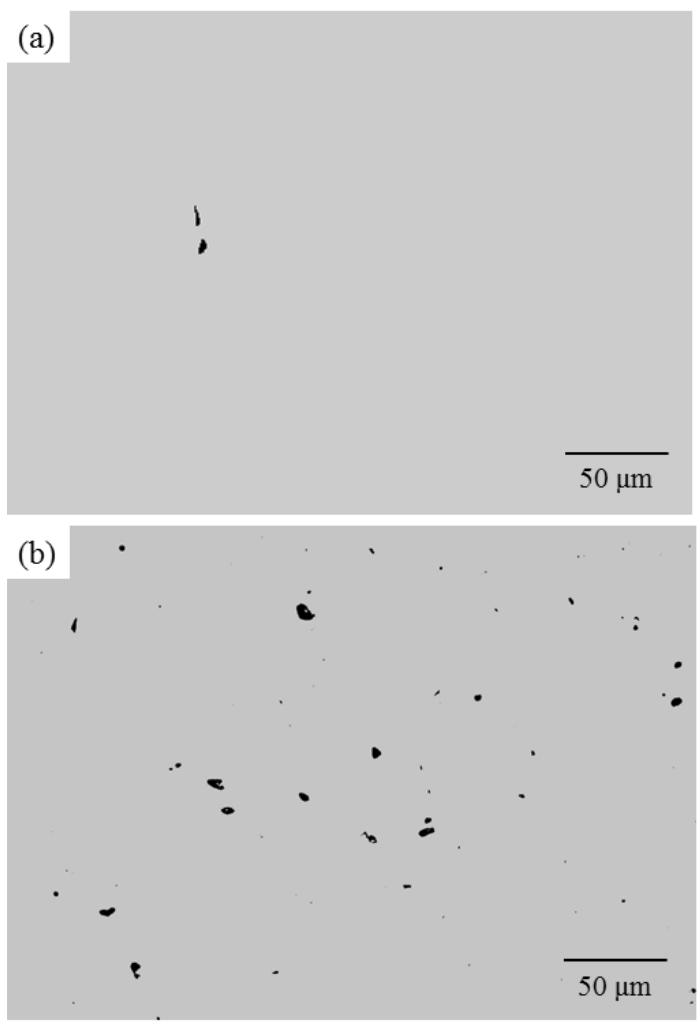

Fig. 3. Optical micrographs of the (a) as-built and (b) heattreated samples. Black pixels indicate voids. induced pores are smaller in size and could have incurred weaker stress concentration under the cyclic loading, such that they were not critical in causing crack initiation for samples in the as-built condition.

Defect condition worsened after heat treatment. As shown in Figure 3b, irregularly-shaped voids are prevalent in the heat-treated sample. While the increase in size of the defects is marginal (from about $5 \mu \mathrm{m}$ to 10 $\mu \mathrm{m})$, there is a significant increase in number. These defects are smaller in comparison with that found at the fatigue fracture origin in Figure 2c. To verify if they are the same type of defects, the fractographs were examined more closely. Similar defects of smaller size were found in the interior of the sample, as shown in Figure 2d. Since fatigue failure seeks the largestoccurring defect, it is possible that the use of optical microscope for inspection had missed detecting the larger defects. Moreover, quantification of three dimensional defect by the two dimensional microscopic method is affected by stereological error. As the defects are flat and thin, polishing on sections perpendicular to the thickness direction could have easily caused them to be removed. Hence, it is possible that the crack-initiating defects are the same type of defect as those shown on the optical micrograph, and are therefore generated by the heat treatment.

This result indicates that the currently tested heat treatment condition produces defects that are detrimental to fatigue failure. Specifically, as the high temperature heat treatment could have caused the annihilation of 
dislocations at the grain boundaries [32] and recrystallization, any remnant intergranular defects were no longer detrimental to cracking. As a result, the thermally-induced defects became operative and initiated fatigue cracks. The possible mechanisms for the formation of the thermally-induced defect is explained in the next section.

\subsection{Mechanisms of defect formation}

For welded austenitic stainless steel structures containing heat affected zones (HAZ), reheating is known to produce intergranular cracking $[33,34]$. The relaxation of residual stress is opposed by the bulk of the grains, leading to the accumulation of intense plastic strain at the grain. When deformation occurs via the sliding of grain boundaries, grain boundary cracks would be formed.

The defects observed in the heat-treated samples could potentially be attributed to such reheat cracking mechanism. Characteristics of the L-PBF stainless steel 316L microstructure render the material susceptible to reheat cracking, as discussed in the following paragraphs.

Metallurgically, the walls of the cellular grains in LPBF stainless steel $316 \mathrm{~L}$ are packed with a high concentration of dislocation network [35]. While the high dislocation density is desirable in terms of creating a strengthening effect by resisting dislocation motion, locally, it could lead to reduced ductility and embrittled cellular grain boundaries, as reflected in the intergranular crack initiation behaviour of the as-built samples. With rising temperature during annealing, residual stress could not be easily released by intragranular slip as it is impeded by the pre-existing dislocation network. The release of residual stress thus occurs intergranularly across the cellular grain boundaries, promoting the formation of cracks.

Mechanically, L-PBF involves rapid solidification which induces high residual stress levels. For materials with solidification microstructures, local variations in residual stress could be present due to difference in material phases and thermal history [36]. As the consolidation process in L-PBF is characterised by competitive grain growth at the solid-liquid interface [37], local differences in stress state are inevitable. Moreover, with successive laser passes, regions of the material experience repeated heating and cooling such that residual stress of different extent is generated along the build direction [38]. Since the relaxation of residual stress during heat treatment is accompanied by elastic recovery of various regions in the bulk material, it promotes the opening of high stress concentration areas [33] such as pre-existing defects, in this case grain boundaries or gas pores. As temperature in the furnace ramped up to the holding temperature during annealing, stress relaxation could have caused intense plastic strain at the tip of the defects and provoked cracking.

Alternatively, the fusion of pre-existing porosity could also have resulted in defects enlargement after heat treatment. However, as the coalescence of pores under such circumstance takes place via the mechanism of surface tension and thermal equilibrium [39], the resulting porosity tends to be spherical in shape, rather than as the flat and irregular defects observed in this work. Nevertheless, it is possible that neighbouring pores could have diffused into the reheat cracks, expanding the space between the cracked surfaces. Adjacent intercellular spaces could also have diffused out and coalesced, contributing volume to the reheat cracks.

\subsection{Influence on high cycle fatigue properties}

Figure 4a shows the S-N curves for the as-built and heattreated samples adapted from the authors' prior work [40]. Heat treatment caused a near parallel downward shift of the S-N curve, where the fatigue lives and fatigue strength of the heat-treated samples are $60 \%$ and $13 \%$ lower than the as-built samples respectively. The fatigue limit, as represented by the stress levels of the runout samples, however are similar regardless of heat treatment.

As besides impacting the crack initiation mechanism via the generation of failure-critical defects, heat treatment also alters the material microstructure which influences the fatigue deformation behaviour, the effects of both factors thus need to be considered for assessing the observed high cycle fatigue properties. For stainless steel $316 \mathrm{~L}$, annealing causes the breaking down of the pre-existing intercellular dislocation network which is instrumental in providing the high strength of the material [35]. For the samples tested in this work, the yield strength of the as-built samples is about $590 \mathrm{MPa}$ and the elongation to failure is $43 \%$; after heat treatment, yield strength dropped by nearly $30 \%$, to $420 \mathrm{MPa}$, while ductility increased by approximately the same extent to $56 \%$ [40].

Since the samples were tested over the same stress range, plastic deformation and the accumulation of plastic strain could have taken place via the ratcheting mechanism in some of the heat-treated samples. To analyse this behaviour, the ratcheting strain $\epsilon_{r}$ was calculated using the relation:

$\epsilon_{r}=0.5\left(\epsilon_{\max }+\epsilon_{\text {min }}\right)$

where $\epsilon_{\max }$ and $\epsilon_{\min }$ are the maximum and minimum strain values of a particular cycle.

Figure $4 \mathrm{~b}-\mathrm{c}$ shows the plot of $\epsilon_{\mathrm{r}}$ against the cycles to failure values of samples tested at maximum applied cyclic stress of $438 \mathrm{MPa}$ and $365 \mathrm{MPa}$ respectively. It can be seen that under the higher stress condition, the asbuilt and heat-treated samples exhibit distinctly different cyclic strain responses. For the as-built sample, strain stabilised after a relatively short life and remained constant throughout before catastrophic failure took place. The heat-treated sample, however, shows continuously increasing strain with the progression of asymmetric cycling. For the lower stress condition, ratcheting strain stabilised for both as-built and heattreated samples, and failure did not occur when the tests were terminated at $10^{6}$ cycles. 
The behaviour of the heat-treated sample under the high stress loading is associated with ratcheting strain accumulation, where under stress-controlled cyclic loading with positive mean stress, inelastic strain builds up with each cycle. Besides fatigue damage, which arose from the alternating stress, permanent deformation in the direction of loading also took place in the bulk material, increasing crack density and promoting necking of the sample. Locally, at the crack-initiating defect, ratcheting can trigger microcrack nucleation and shorten the fatigue initiation life [41]. As heat treatment caused significant strength degradation, the applied cyclic stress exceeded the yielding stress, giving rise to the static deformation mode.

It is interesting to note that in comparison with the as-built condition, heat treatment incurred much lower reduction in fatigue strength $(13 \%)$ than yield strength $(30 \%)$, and most of the heat-treated samples survived long lives, i.e. $>10^{5}$ cycles, despite the ratcheting mechanism. This could be attributed to the higher ductility after heat treatment, which partially offset the effect of strength reduction on fracture toughness and allowed the material to sustain greater damage prior to fracture.

(a)

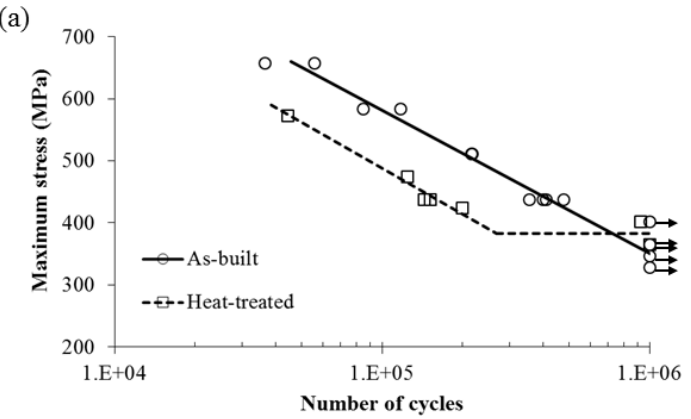

(b)

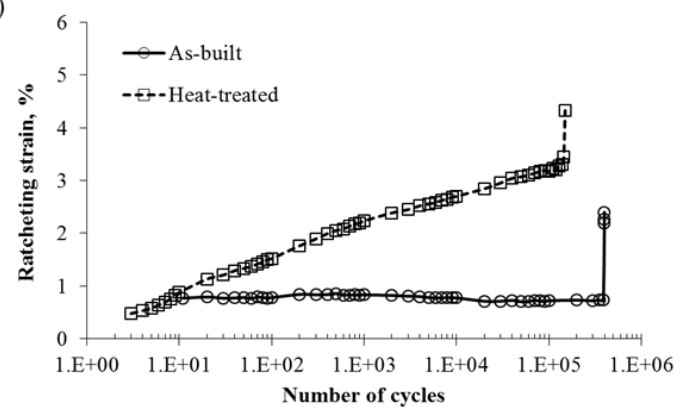

(c)

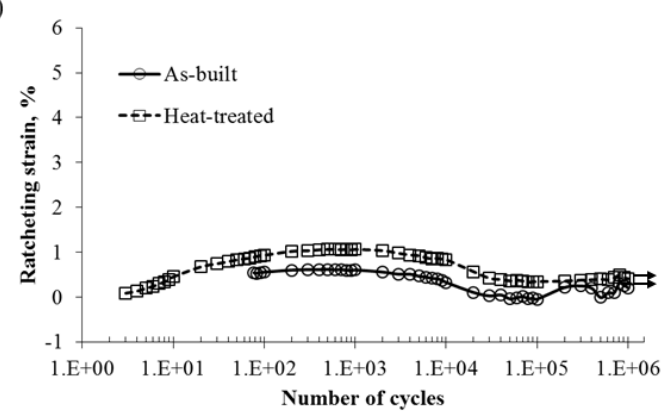

Fig. 4. (a) S-N curves; ratcheting strain against number of cycles for samples tested at maximum applied cyclic stress of (b) $438 \mathrm{MPa}$ and (c) $365 \mathrm{MPa}$.
When the cyclic stress is lower than the yield strength, strain accumulation did not take place for both the as-built and heat-treated samples as global deformation is mostly in the elastic domain. This explains the similar fatigue limits in spite of heat treatment. In fact, crack growth experiments done by Riemer et al. [26] showed that L-PBF stainless steel $316 \mathrm{~L}$ subjected to high temperature thermal treatment in the form of HIP has better near threshold performance because of coarsened grains, which act as obstacles to crack growth. Hence, it is expected that the long life fatigue properties of the heat-treated material are to be comparable, if not superior to the as-built form.

In summary, high temperature heat treatment of LPBF stainless steel $316 \mathrm{~L}$ generates critical fatigue failure defects and microstructure transformation, both of which affect the high cycle fatigue properties. In the finite life regime, fatigue deformation is complemented by ratcheting strain accumulation as a result of the reduced strength after heat treatment; the effect of crack initiating defects could play a secondary role in altering the fatigue stress-life relation since failure-critical defects are present in both the as-built and the heat-treated forms. In the low stress high cycle regime, where deformation is dominated by fatigue activities, the fatigue properties of both types of samples converge, leading to similar long life performance. Further experiments could be performed to evaluate the effect of thermally-induced defect and microstructure on the fatigue crack initiation and failure behaviours in the very high cycle fatigue regime.

\section{Conclusions}

The high cycle fatigue properties and crack initiation behaviours of L-PBF stainless steel $316 \mathrm{~L}$ in the as-built and solution-annealed conditions were examined in this work. Based on the experimental results, it can be concluded that:

1. High temperature annealing leads to the formation of thermally-induced defects which become operative under high cycle fatigue loading and cause fatigue crack initiation.

2. The thermally-induced defects could have been formed due to reheat cracking during residual stress relaxation. The diffusion and coalescence of neighbouring porosities and intercellular spaces could also have led to the enlargement of the reheat cracks. The critical annealing conditions, in terms of temperature and duration, leading to the formation of the defects remain to be further investigated.

3. The annealing condition tested in this work produces simultaneous effects on crack initiation and the bulk deformation behaviour. Fatigueratcheting interactions were responsible for the fatigue properties of the heat-treated samples under the tested stress condition and the reduced fatigue resistance. Better fatigue properties are expected for the heat-treated samples in the long life regime due to reduced intergranular brittleness and coarsened grains which impede crack growth. 


\section{Acknowledgement}

This work was supported by the Singapore Economic Development Board (EDB) Industrial Postgraduate Programme (IPP).

\section{References}

1. C. Yan, L. Hao, A. Hussein, and D. Raymont, Int. J. Mach. Tools Manuf., 62, 32-38 (2012)

2. P. Razvan and P. Ancuta, New Trends in $3 D$ Printing, (InTech, 2016).

3. M. Wong, I. Owen, C.J. Sutcliffe, and A. Puri, International Journal of Heat and Mass Transfer, 52(1), 281-288 (2009)

4. B. Vandenbroucke and J.-P. Kruth, Rapid Prototyping Journal, 13(4), 196-203 (2007)

5. M. Javaid and A. Haleem, Alexandria Journal of Medicine, (2017)

6. P. Hanzl, M. Zetek, T. Bakša, and T. Kroupa, Procedia Engineering, 100, 1405-1413 (2015)

7. K. Guan, Z. Wang, M. Gao, X. Li, and X. Zeng, Mater. Des., 50, 581-586 (2013)

8. G. Yu, D. Gu, D. Dai, M. Xia, C. Ma, and K. Chang, Appl. Phys. A, 122(10), 891 (2016)

9. M. Gorelik, Int. J. Fatigue, 94, 168-177 (2017)

10. X. Huang, M.C. Chaturvedi, and N.L. Richards, Metall. Mater. Trans. A, 27(3), 785-790 (1996)

11. M.H. Bina, Metallurgy - Advances in Materials and Processes, (InTech, Rijeka. 2012).

12. P. Mercelis and J.-P. Kruth, Rapid Prototyping Journal, 12(5), 254-265 (2006)

13. L. Parry, I.A. Ashcroft, and R.D. Wildman, Additive Manufacturing, 12, 1-15 (2016)

14. K. Kempen, B. Vrancken, L. Thijs, S. Buls, J. Van Humbeeck, and J.-P. Kruth. in Solid Freeform Fabrication Symposium Proceedings. 2013)

15. J.J. Lewandowski and M. Seifi, Annu. Rev. Mater. Res, 46, 14.1-14.36 (2016)

16. V. Cain, L. Thijs, J. Van Humbeeck, B. Van Hooreweder, and R. Knutsen, Additive Manufacturing, 5, 68-76 (2015)

17. T. Vilaro, C. Colin, J.D. Bartout, L. Nazé, and M. Sennour, Mater. Sci. Eng. A, 534, 446-451 (2012)

18. E. Wycisk, A. Solbach, S. Siddique, D. Herzog, F. Walther, and C. Emmelmann, Physics Procedia, 56, 371-378 (2014)

19. T.M. Mower and M.J. Long, Mater. Sci. Eng. A, 651, 198-213 (2016)

20. A. Yadollahi, N. Shamsaei, S.M. Thompson, A. Elwany, and L. Bian, Int. J. Fatigue, (2016)

21. D. Greitemeier, F. Palm, F. Syassen, and T. Melz, Int. J. Fatigue, 94, 211-217 (2017)

22. G. Kasperovich and J. Hausmann, J. Mater. Process. Technol., 220, 202-214 (2015)

23. V. Popovich, E. Borisov, A. Popovich, V.S. Sufiiarov, D. Masaylo, and L. Alzina, Mater. Des., 131, 12-22 (2017)

24. Y. Bai, Y. Yang, D. Wang, and M. Zhang, Mater. Sci. Eng. A, (2017)
25. M.M. Dewidar, K.A. Khalil, and J. Lim, Transactions of Nonferrous Metals Society of China, 17(3), 468-473 (2007)

26. A. Riemer, S. Leuders, M. Thöne, H. Richard, T. Tröster, and T. Niendorf, Eng. Fract. Mech., 120, 15-25 (2014)

27. S. Leuders, T. Lieneke, S. Lammers, T. Tröster, and T. Niendorf, J. Mater. Res., 29(17), 1911-1919 (2014)

28. P.J. da Silva Bartolo, A.C.S. de Lemos, A.M.H. Pereira, A.J.D.S. Mateus, C. Ramos, C. Dos Santos, D. Oliveira, E. Pinto, F. Craveiro, and H.M.C. da Rocha Terreiro. in Proceedings of the 6th International Conference on Advanced Research in Virtual and Rapid Prototyping. (Portugal, 2013)

29. Y. Zhong, L. Liu, S. Wikman, D. Cui, and Z. Shen, J. Nucl. Mater., 470, 170-178 (2016)

30. M. Zhang, C.-N. Sun, X. Zhang, P.C. Goh, J. Wei, D. Hardacre, and H. Li, Mater. Sci. Eng. A, 703, 251-261 (2017)

31. B. Zhang, Y. Li, and Q. Bai, Chinese Journal of Mechanical Engineering, 30(3), 515-527 (2017)

32. K. Saeidi, X. Gao, Y. Zhong, and Z.J. Shen, Mater. Sci. Eng. A, 625, 221-229 (2015)

33. A. Vinckier and A. Dhooge, J. Heat. Treat., 1(1), 7280 (1979)

34. R. Skelton, I. Goodall, G. Webster, and M. Spindler, Int. J. Press. Vessels Pip., 80(7), 441-451 (2003)

35. L. Liu, Q. Ding, Y. Zhong, J. Zou, J. Wu, Y.-L. Chiu, J. Li, Z. Zhang, Q. Yu, and Z. Shen, Mater. Today, (2017)

36. P.J. Withers and H.K.D.H. Bhadeshia, Mater. Sci. Technol., 17(4), 366-375 (2001)

37. A.R.A. Dezfoli, W.-S. Hwang, W.-C. Huang, and T.-W. Tsai, Scientific Reports, 7, 41527 (2017)

38. Y. Liu, Y. Yang, and D. Wang, Int. J. Adv. Manuf. Technol., 87(1-4), 647-656 (2016)

39. H. Zhao and T. DebRoy, Welding Journal, 80(8), 204-210 (2001)

40. M. Zhang, C.-N. Sun, X. Zhang, P.C. Goh, J. Wei, H. Li, and D. Hardacre, JOM, 70(3), 390-395 (2018)

41. P. Lukáš and L. Kunz, Int. J. Fatigue, 11(1), 55-58 (1989) 